\title{
Expression cloning of new receptors used by simian and human immunodeficiency viruses
}

\section{HongKui Deng*, Derya Unutmaz ${ }^{\star} \dagger$, Vineet N. KewalRamani \& Dan R. Littman $\dagger$}

$\dagger$ Division of Molecular Pathogenesis, Skirball Institute of Biomolecular Medicine, Howard Hughes Medical Institute, New York University Medical Center, 540 First Avenue, New York, New York 10016, USA

* These authors contributed equally to this work.

Several members of the chemokine-receptor family serve, in conjunction with $\mathrm{CD} 4$, as receptors for the entry of human immunodeficiency virus type I (HIV-1) into cells ${ }^{1-6}$. The principal receptor for entry of macrophage-tropic (M-tropic) HIV-1 strains is CCR5, whereas that for T-cell-line-tropic (T-tropic) strains is CXCR4. Unlike HIV-1, infection with either M-tropic or T-tropic strains of simian immunodeficiency virus (SIV) can be mediated by CCR5, but not CXCR4 (refs 7-10). SIV strains will also infect $\mathrm{CD}^{+}$cells that lack CCR5, which suggests that these strains use as yet unidentified receptors ${ }^{7,9,10}$. Here we use an expression-cloning strategy to identify SIV receptors and have isolated genes encoding two members of the seven-transmembrane G-protein-coupled receptor family that are used not only by SIVs, but also by strains of HIV-2 and M-tropic HIV-1. Both receptors are closely related to the chemokine-receptor family and are expressed in lymphoid tissues. One of the receptors is also expressed in colon and may therefore be important in viral transmission. Usage of these new receptors following experimental infection of non-human primates with SIV strains may provide important insight into viral transmission and the mechanisms of SIV- and HIV-induced acquired immune-deficiency syndrome.

To identify the receptors used by SIV, we used an HIV-luciferase vector pseudotyped with SIV envelope glycoproteins (Env) to infect several $\mathrm{CD}^{+}{ }^{+}$cell lines ${ }^{2}$. A murine 3T3.CD4 cell line expressing CCR5, but not CXCR4, was readily infected with viruses bearing Env proteins from either Rhesus macaque or African green monkey strains ( $\mathrm{SIV}_{\text {maclA11 }}$ (ref. 11) or $\mathrm{SIV}_{\text {agmTY01 }}$ (ref. 12), respectively) (Fig. 1a). The human astroglial U87.CD4 cell line, which does not express CCR5 or CXCR4 and is resistant to infection with pseudotypes displaying T-tropic HXB2 or M-tropic JRFL Env glycoproteins ${ }^{13}$, was readily infectable with $\mathrm{SIV}_{\text {agmTY01 }}$ and, to a lesser degree, with SIV mac1A11 Env-pseudotyped viruses (Fig. 1b). In contrast, CEM $\times 174$, a T-cell/B-cell hybrid that does not express CCR5 either and is commonly used to amplify SIV in culture ${ }^{14}$, was

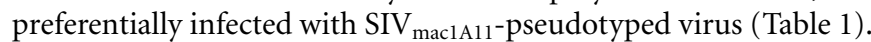
The pattern was similar in the Hut78 T-cell line (Table 1), suggesting the presence of at least two additional receptors for SIV.

To isolate the genes encoding such receptors, we used an expression-cloning strategy (Fig. 2a). A human T-cell clone cDNA library, subcloned in the retroviral vector pMX (obtained from T. Kitamura, DNAX), was transfected into BOSC23 packaging cells and the supernatant from these cells was then used to infect 3T3.CD4 cells. The transduced 3T3.CD4 cells were in turn challenged with a selectable replication-defective virus (HIV-puro) pseudotyped with either the SIV agmTY01 or SIV mac1A11 Env glycoproteins, followed by puromycin selection. A total of 280 puromycin-resistant colonies were obtained with SIV $_{\text {agmTY01 }}$ infection and about 170 with $\mathrm{SIV}_{\text {mac1A11 }}$ infection. These colonies were then tested for their ability to be infected with HIV-luc pseudotyped with the HIV-1 M-tropic Env protein JRFL. Representative results after infection of several

candidate colonies with virus bearing SIV or HIV Env glycoproteins

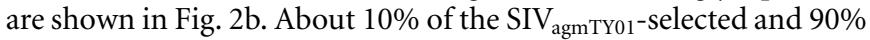

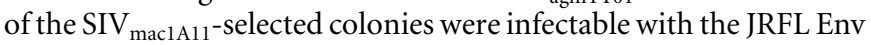
pseudotypes, and all were shown by polymerase chain reaction (PCR) and antibody staining to express CCR5 (for example, clones C1.193 and C2.219 in Fig. 2b). Genomic DNAs extracted from CCR5-negative colonies were subjected to PCR amplification using the primers flanking inserts in the retroviral vectors. A common 2.1kilobase $(\mathrm{kb})$ band was detected with $\mathrm{SIV}_{\text {agmTY01-selected colonies, }}$

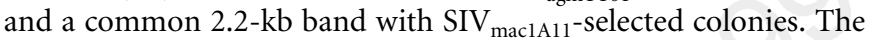
PCR products were subcloned into expression vector pCR3.1 (Invitrogen) and the DNA sequenced. The complementary DNA selected with the SIV agmTY01 pseudotypes was found to encode a new protein of 342 amino acids, which we named Bonzo, whereas its counterpart selected with the $\mathrm{SIV}_{\text {mac1A11 }}$ pseudotypes encodes a protein of 360 amino acids, designated BOB (for brother of Bonzo). Comparison of these sequences with those in genome databases indicates that both molecules are members of the large family of Gprotein-coupled receptors. BOB is identical to a previously cloned orphan receptor, GPR15 (ref. 15), whereas Bonzo has no identity to

\begin{tabular}{lccc}
\hline Table 1 Infection of T-cell lines with SIV isolates \\
\hline & \multicolumn{3}{c}{ Luciferase activity (c.p.s. $\times 10^{-3}$ ) } \\
\cline { 2 - 4 } Cell line & No Env & mac1A11 & agmTYO1 \\
CEM & $<1$ & 21 & 736 \\
CEM $\times 174$ & $<1$ & 2,025 & 191 \\
Hut78 & $<1$ & 145 & 51
\end{tabular}

Infection of transformed $\mathrm{CD}^{+}$cell lines was done by using HIV-luciferase-reporter virus pseudotyped with SIV Env proteins. Values are the average of duplicate determinations. Standard errors were less than $15 \%$ of the average value.
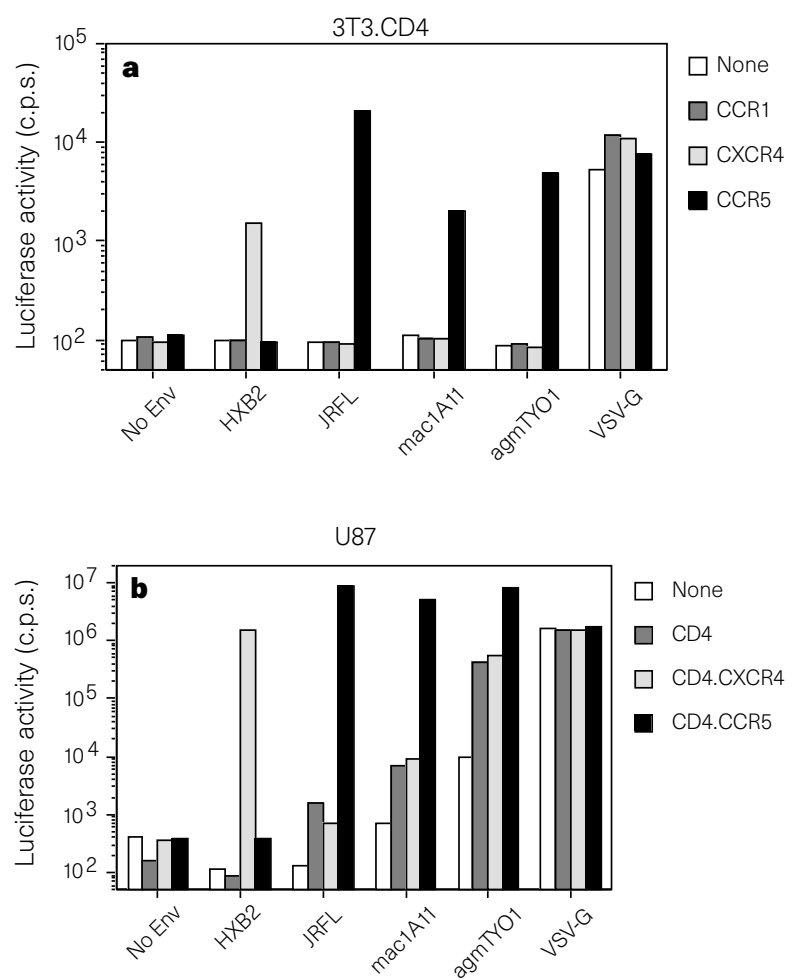

Figure 1 Specificity of SIV Envs for chemokine receptors on target cells. a, Infection of 3T3.CD4 cells stably expressing CCR1, CCR5, or CXCR4 with luciferase reporter viruses pseudotyped with Env proteins of HIV-1 (HXB2 and JRFL), SIV (mac1A11 and agmTY01) or VSV-G. b, Infection of U87 cells and U87.CD4 cells with the pseudotyped reporter viruses. Lysates were assayed 3 days post-infection for luciferase activity as described in Methods. 
sequences in the expressed-sequence tag (EST) databases. Both BOB and Bonzo are related to chemokine-receptor family proteins but share only $25-30 \%$ amino-acid sequence identity with CCR5 and CXCR4. Sequence alignments of these receptors and of CCR5 and CXCR4 are shown in Fig. 2c. Using specific primers, we also cloned homologues of the SIV receptors from African green monkey and pigtail macaque. These were found to be $94-97 \%$ identical to the human sequences (data not shown; sequences are lodged with Genbank).

To test the viral-receptor function of the newly identified molecules, their cDNAs were cloned into the pBABE-puro expression vector and were transiently transfected into human 293T cells together with human CD4. Cells were separately transfected with vectors encoding CCR 5 or CCR 1 as controls. Transfected cells were then infected with HIV-luc viruses pseudotyped with Env glycoproteins from a diverse set of SIV, HIV-2 and primary HIV-1 isolates. As shown in Fig. 3a, the three SIV envelopes tested were all able to use Bonzo, BOB and CCR5. The inability of SIV Envpseudotyped virus to infect CCR1-transfected control cells also shows that none of the SIV Env glycoproteins tested can use CXCR4, which is expressed endogenously in 293T cells. Most of the HIV-2 Env proteins tested were also able to use $\mathrm{BOB}$ and/or Bonzo (Fig. 3a). Several HIV-2 isolates that grow on transformed T-cell lines can also use CXCR4, explaining their high background in 293T cells (for example, ROD and UC2). Consistent with our previous results $^{10}$, most of the HIV-2 Env proteins tested were able to use CCR5 to varying degrees, with the exception of UC2, which uses CXCR4 exclusively. The specificities of the different HIV-2 Env proteins have been confirmed in infections of 3T3.CD4 cells, as discussed later (Fig. 3c, and data not shown).

We next tested a panel of HIV-1 Env glycoproteins whose tropism for CCRs and CXCR4 had been determined. Several CCR5-tropic
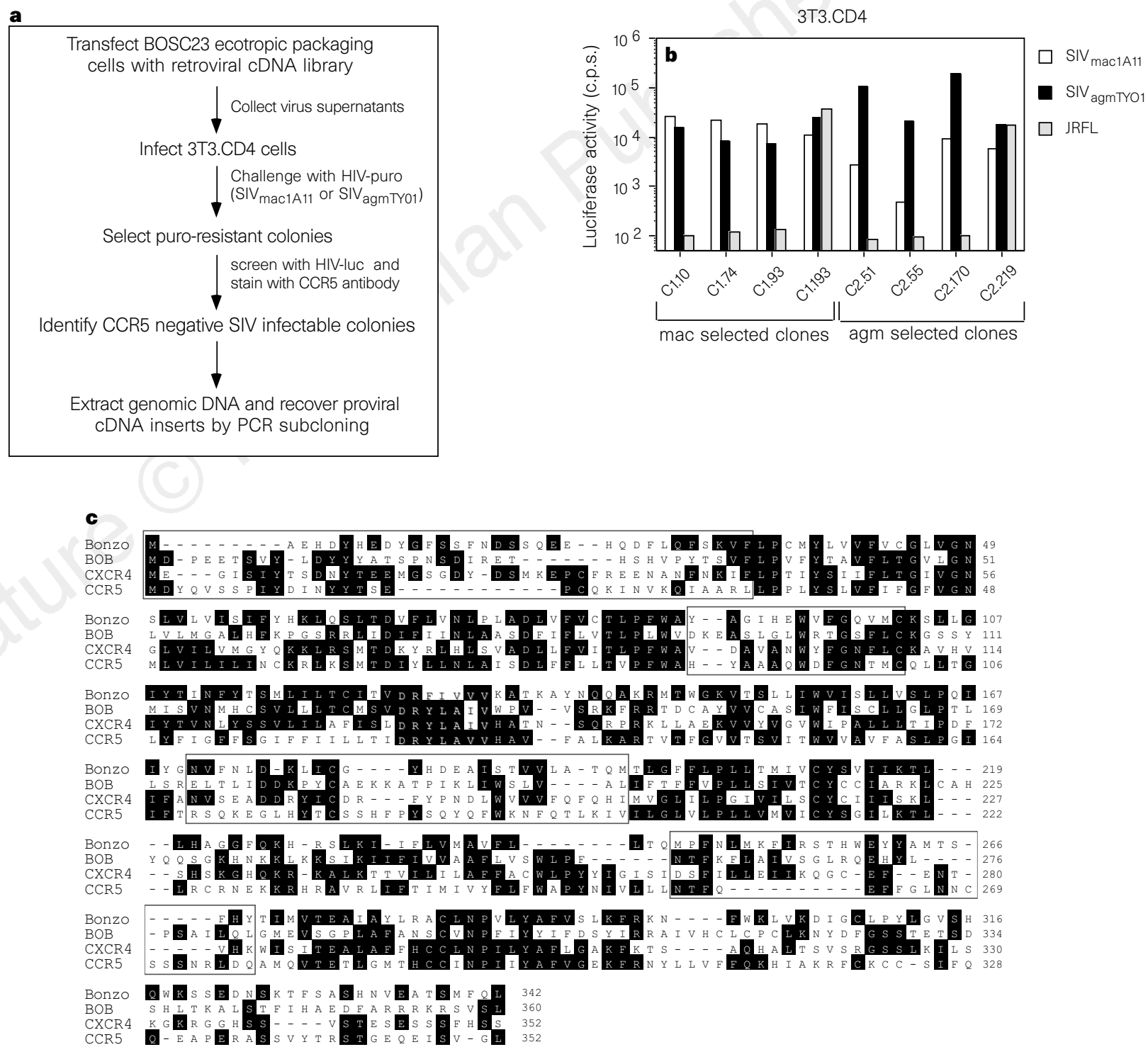

Figure 2 Expression cloning of novel SIV receptors. a, Outline of the expression cloning strategy used to isolate SIV receptors. b. Infection of representative 3T3.CD4 clones, previously selected with HIV-puro(SIV) pseudotyped virus, with HIV-luc pseudotyped with SIV and M-tropic HIV-1 Env proteins. c, Amino-acid sequence alignment of the receptors encoded by the BOB and Bonzo cDNAs with HIV receptors CXCR4 and CCR5. Identical amino acids are shaded and the putative extracellular domains (based on CCR5 alignment) are boxed. The 'DRY box' sequence, which is involved in signalling and is characteristic of chemokine receptors, is highlighted in bold. Bonzo contains a divergent 'DRY box' motif compared to other HIV receptors; this sequence has thus been observed in only one other chemokine receptor, EBI 2. 


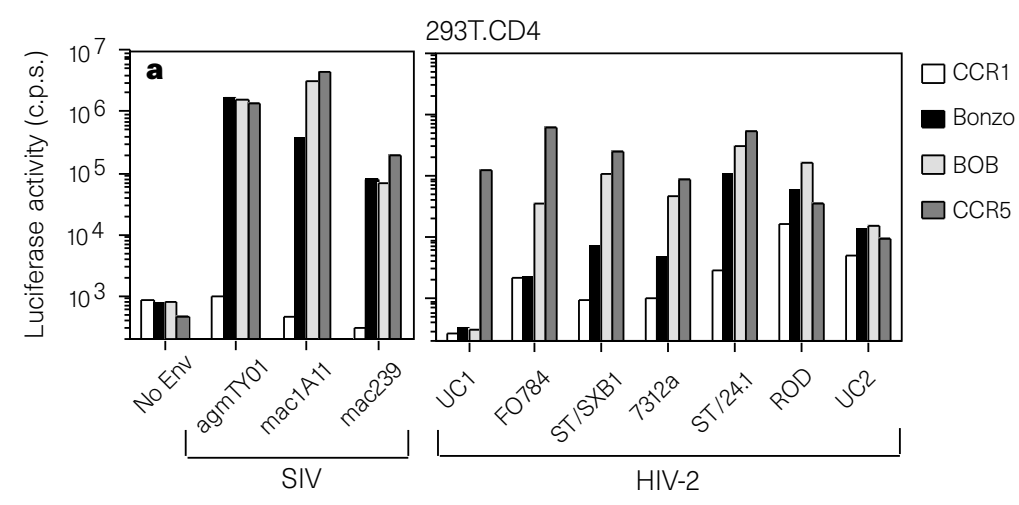

Figure $3 \mathrm{BOB}$ and Bonzo mediate entry of SIV, HIV-2 and Mtropic or dual-tropic HIV-1 strains. a, Infection of 293T cells transiently cotransfected with plasmids encoding CD4 plus CCR1, Bonzo, BOB or CCR5. The cells were infected 2 days later with luciferase-reporter viruses pseudotyped with different SIV or HIV-2 Env proteins. The HIV-2 Env proteins, UC1, FO784, ST/SXB1 and 7312a have been shown to use CCR5; ST/24.1 and ROD can use both CCR5 and CXCR4; UC2 uses only CXCR4. b, Infection of the transiently transfected 293T cells with HIV-luc pseudotyped with HIV-1 Env proteins or control VSV-G. Target cells expressing CXCR4 were also included to identify T-tropic Env proteins, even though 293T cells express endogenous CXCR4. c, 3T3.CD4 cells stably expressing CCR1, BOB, Bonzo, CCR5 or CXCR4 were infected with the luciferase reporter viruses as described. Luciferase activity was measured on day 3 . Similar results were obtained in three independent experiments.
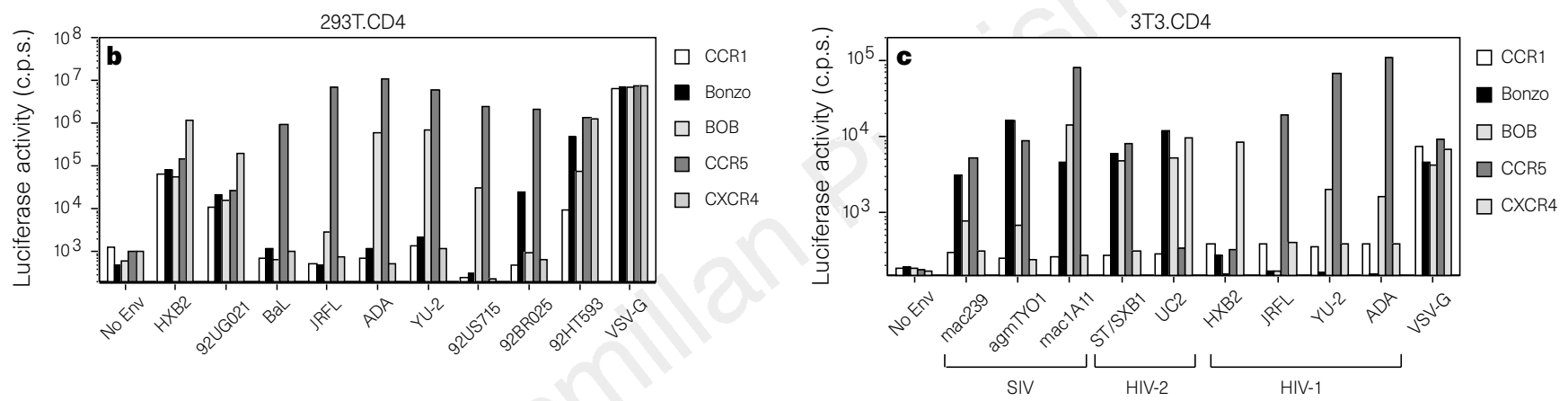

Env glycoproteins (ADA and YU-2, which can also use CCR3 (refs 6, 16), 92US715 and 91US005) and one dual-tropic for CXCR4 and R5 (92HT593) were also able to infect cells transfected with BOB (Fig. 3b, and data not shown). Two of the Env proteins from primary HIV-1 strains (the dual tropic 92HT593 and the CCR5-tropic 92BR025) also utilized Bonzo (Fig. 3b). None of the three T-tropic envelopes tested (HXB2, 92UG021 or 92HT599) was found to use BOB or Bonzo. Several other CCR5-tropic Env proteins (BaL, 92UG975, 93HT966, 92RW020, 93TH976, 92BR020 and 93MW965) did not use these receptors either, and one (JRFL) used BOB only weakly (Fig. 3b, and data not shown).

Infection of 293T.CD4 cells with viruses using CXCR4 results in a high background owing to expression of endogenous CXCR4 (for example, HIV-2 UC2 in Fig. 3a). We therefore stably introduced Bonzo, BOB, CCR5 and CCR1 into mouse 3T3 cells expressing human CD4, and infected these with the panel of HIV-luc pseudotype viruses (Fig. 3c). The infection results were consistent with those obtained from the transient transfections of $293 \mathrm{~T}$ cells. The

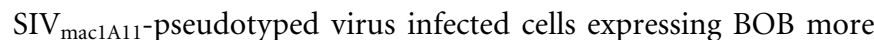
effectively than cells expressing Bonzo, whereas the converse was observed with virus bearing SIV $_{\text {agmTY01 }}$ Env. Infection of human osteosarcoma cells, expressing CD4 and either BOB, Bonzo or CCR5, with replication-competent $\mathrm{SIV}_{\mathrm{agm}}$ and $\mathrm{SIV}_{\text {mac }}$ strains yielded similar results (data not shown).

We next determined the expression pattern of the newly identified receptors in normal human tissues using northern blot hybridization (Fig. 4a). Bonzo cDNA hybridized to a 2.1-kb mRNA species expressed at high levels in spleen, thymus, small intestine and, to a lesser extent, in peripheral blood leukocytes (PBL), prostate and colon. In the placenta, a $2.6-\mathrm{kb}$ species was detected; whether this represents a different homologous gene product, the product of differential splicing, or an antisense messenger RNA is unknown. Similarly, BOB mRNA was detected in spleen and PBL; expression was weaker in thymus and small intestine. BOB mRNA

was present in colon, where its high expression indicates that it may be expressed in non-lymphoid cells. It will therefore be important to determine whether this expression pattern is relevant for sexual transmission of HIV.

Expression of the newly identified receptors was also analysed in peripheral blood mononuclear cells (PBMC) and in FACS-purified subsets by PCR with reverse transcription (RT-PCR) (Fig. 4b). Expression of Bonzo mRNA was evident in unstimulated and phytohaemagglutinin (PHA)-stimulated PBMC, T cells and monocytes, but not in B cells. BOB mRNA was detected in PHAstimulated PBMC, purified T cells, and weakly in unstimulated PBMC. However, expression was either minimal or absent in monocytes and B cells.

We next investigated whether susceptibility of various cell lines to infection with SIV could be explained by the expression of the new receptors. Northern blot analysis of RNA from cell lines shows that $\mathrm{CEM} \times 174$ cells, the parental 174 cells, and Hut78 cells express high levels of BOB but not of Bonzo (Fig. 4c). This pattern of expression correlates with the preferential infection of CEM $\times 174$ and Hut78 cells by the SIVs from rhesus macaque. Low levels of Bonzo mRNA could be detected in CEM and U87 cells, both by northern blotting (Fig. 4c) and by RT-PCR (data not shown). Furthermore, antiserum raised against the N-terminal sequence of Bonzo blocked infection of U87.CD4 and CEM cells with HIV-luc pseudotyped with SIV Env glycoproteins (data not shown). The expression pattern of Bonzo thus probably explains the ability of these cells to be infected with African green monkey strains of SIV.

In humans, CCR5-tropic viruses are primarily involved in transmission, whereas viruses with broader tropism, particularly for CXCR4, emerge during the progression to immunodeficiency ${ }^{17}$. It is not yet known whether appearance of CXCR4-tropic viruses is a consequence or cause of the decline of the immune system. Insight into this key problem of virus evolution is likely to require experimentation in animal models. Infection of non-human pri- 

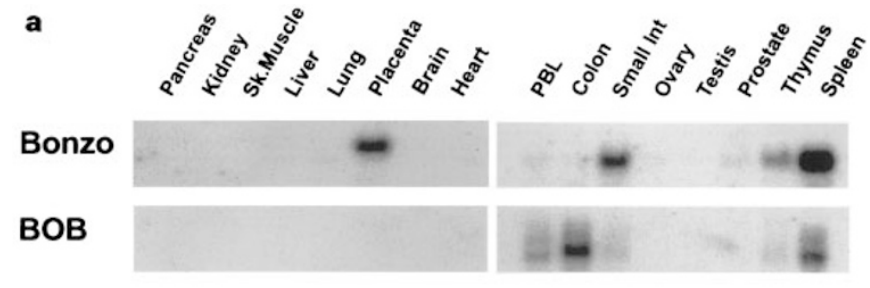

b

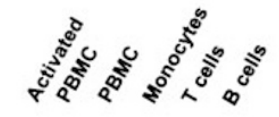

C

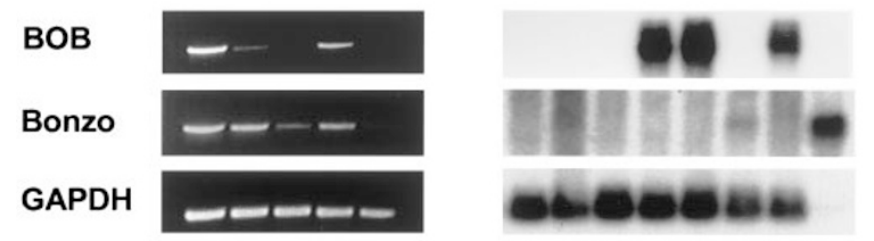

Figure 4 Expression of $B O B$ and Bonzo mRNAs in human lymphoid tissues and cell lines. a, RNA tissue blot of various human tissues (Clontech) probed with fulllength $\mathrm{BOB}$ and Bonzo cDNAs as described in Methods. The signals correspond to 2.1-kb Bonzo and 2.2-kb BOB transcripts. b, RT-PCR analysis of BOB and Bonzo transcripts in human PBMC, resting or stimulated with PHA (Sigma) for 7 days, and purified lymphocyte subsets. Total RNAs isolated from these cells were amplified with primers specific for BOB, Bonzo or glyceraldehyde-3-phosphate dehydrogenase (GAPDH) as a control (bottom). Primer specificity has been verified on 3 T3 cells expressing $\mathrm{BOB}$ or Bonzo. c. Northern blot analysis of poly $(A)^{+}$mRNA from different cell types. Each lane was loaded with $5 \mu \mathrm{g}$ poly $(A)^{+}$ mRNA, except for the PBMC lane, which contained $10 \mu \mathrm{g}$ total RNA.

mates with SIV is currently the only good animal model for studying the pathogenesis of the immunodeficiency viruses ${ }^{18}$. Moreover, different species of non-human primates vary widely in their responses to SIV infection. For example, Rhesus macaques succumb to immunodeficiency that closely resembles AIDS in humans, but sooty mangabeys and African green monkeys can sustain infection with little evidence of immune-system damage ${ }^{19}$. These interspecies differences may provide important clues for understanding and containing disease progression in HIV-infected humans. Although SIV viruses do not seem to use CXCR4, they use CCR5, Bonzo and $\mathrm{BOB}$ as receptors. Variations in the structure or expression of these molecules may represent host variables that influence the outcome of SIV infection. It will therefore be important to determine whether the ability of primary strains of SIV to use the newly described receptors correlates with the susceptibility of animals to progressive disease.

The finding that envelope glycoproteins from both HIV-1 and HIV-2 primary strains can use Bonzo and $\mathrm{BOB}$ as receptors raises the possibility that these receptors may be important in infection of humans. Recent reports have also described CCR $5^{-/-}$homozygous individuals who are infected with HIV-1 (refs 20-22). As several Mtropic strains can efficiently use the new receptors, especially $\mathrm{BOB}$, these receptors may substitute for CCR5 in establishing HIV-1 infection. However, these individuals may be infected with CXCR4tropic viruses. SIV can replicate in human CCR5-deficient $\mathrm{PBMC}^{7}$, which on the basis of our results can be explained by the ability of SIVs to use Bonzo and/or BOB as receptors in the mutant cells; it will be important to determine whether HIV-1 recovered from infected CCR5-negative individuals can also use these newly identified receptors.

There are now six members of the chemokine-receptor family that function in HIV-1 entry: CCR5, CXCR4, CCR2b, CCR3, Bonzo and BOB. Application of the expression-cloning approach described here should identify additional receptors if primary HIV-1 Env glycoproteins and expression libraries from other tissues are used in the screening. The ability of HIV s to infect cells by way of the newly described receptors, which are expressed in human $\mathrm{T}$ lymphocytes and monocytes, may complicate strategies aimed at blocking CCR5-tropic virus entry. To understand the role of these receptors in infection and in the progression of disease will require the ligands for Bonzo and $\mathrm{BOB}$ to be identified and the biological functions of the receptors to be elucidated.

Note added in proof: Since this manuscript was submitted, S. Liao et al. ${ }^{31}$ have reported on HIV-1 receptor, STRL33, which is the same as Bonzo.

\section{Methods}

Expression cloning. A human T-cell clone cDNA library L10/MX, in the retroviral vector pMX, was provided by $\mathrm{T}$. Kitamura (DNAX). High-titre retroviruses, produced by transient transfection of the library into the packaging cell line BOSC23 (ref. 23), were used to infect NIH3T3 cells stably expressing human CD4. Briefly, $10 \mu \mathrm{g}$ retroviral plasmid DNA was transfected into BOSC 23 cells $\left(2 \times 10^{6}\right.$ cells in a $10-\mathrm{cm}$ culture dish) by calcium phosphate precipitation. Two days post-transfection, the culture supernatant was cleared of cell debris and added with $8 \mu \mathrm{g} \mathrm{ml}^{-1}$ polybrene to ten $10-\mathrm{cm}$ dishes, each containing $5 \times 10^{5} 3 \mathrm{~T} 3$.CD4 cells. After $16 \mathrm{~h}$ incubation at $37^{\circ} \mathrm{C}$, the viral supernatant was replaced with DMEM containing $10 \%$ fetal calf serum. This high multiplicity of infection resulted in 100\% transduction of the 3T3.CD4 cells, as assayed in controls in which pMX.mCD4, whose product is detectable by FACS analysis, was mixed in various ratios with the cDNA library. The library-transduced 3T3.CD4 cells were then transferred to $15-\mathrm{cm}$ plates and infected with a selectable replication-defective virus, HIV-puro, pseudotyped with either $\mathrm{SIV}_{\text {agmTY01 }}$ or SIV mac1A11 Env proteins. HIV-puro pseudotypes were generated by transfecting $293 \mathrm{~T}$ cells with $10 \mu \mathrm{g}$ pHIV-puro (an HIV construct in which env contains a frameshift mutation and nef has been replaced by the SV40 promoter regulating the puromycin-resistance gene, kindly provided by R. Sutton) and $10 \mu \mathrm{g}$ Env-expressing plasmids. Virus-containing supernatants were collected $48 \mathrm{~h}$ post-transfection, filtered, and applied to the librarytransduced 3T3.CD4 cells ( $10 \mathrm{ml}$ viral supernatant per plate). After two days, the 3T3.CD4 cells were replated in 24-well plates $\left(1 \times 10^{5}\right.$ cells per well) with medium containing $5 \mu \mathrm{g} \mathrm{ml}^{-1}$ puromycin. Selection medium was changed every 2 to 3 days, and visible colonies arose typically in 6 to 9 days. These colonies were then tested for their ability to be infected with HIV-luc pseudotyped with the HIV-1 M-tropic Env JRFL or SIV Env proteins from mac1A11 or agmTY01. Colonies infected by JRFL pseudotyped viruses were also stained with anti-CCR5 antibody (gift from C. Mackay).

Genomic DNA isolation and PCR cloning. To recover retrovirus-transduced cDNAs, 50 ng genomic DNA isolated from each puromycin-resistant clone was subjected to PCR. cDNA segments were amplified by using primers complementary to retroviral vector flanking the inserts (upstream, 5' GGTGGACCATTCTCTAGACT; downstream, 5'-CCCTTTTTCTGGAGACTAAAT). The PCR was set up using the Expand kit from BoehringerMannheim and run for 35 cycles at $58^{\circ} \mathrm{C}$ annealing temperature. The resulting PCR fragment was purified and cloned into the pCR3.1 vector (Invitrogen) and sequenced. Amino-acid sequence alignments were analysed using the DNASTAR program.

Pseudotyped virus infection assays. Luciferase reporter viruses were prepared in $293 \mathrm{~T}$ cells as described ${ }^{2,24}$ using pNL-Luc-E $\mathrm{R}^{-}$vector and Env expression plasmids. Env expression vectors for HIV-1 JRFL, ADA, BaL, HXB2, 92UG021, 92US715, 92BR025, 92HT593, as well as SIV mac239, mac1A11 and agmTY01 have been described ${ }^{11,12,24-27}$. The HIV-2 ST24.1 and p7312A envelope expression plasmids were generated by PCR subcloning of the proviral DNA template (gifts from B. Hahn) into the expression plasmid pSP272. The HIV-2 UC1 and UC2 (ref. 28) envelope expression plasmids were from C. Weiss. Proviral DNAs from YU-2, HIV-2 ST/SXB1 (ref. 29) and FO784 (ref. 30) were used in cotransfection with pNL-Luc- $\mathrm{E}^{-} \mathrm{R}^{-}$vector to generate mixed viral particles containing both wild-type and luciferase-containing particles. Pseudotyped viruses in supernatants of transfected 293T cells were quantified by p24 ELISA (Coulter) to normalize virus stocks for infection. 293T cells transiently cotransfected with pMX-CD4 and with pBABE-puro expressing 
CCR1, BOB, Bonzo, CCR5 or CXCR4 (ref. 2) were infected with pseudotyped viruses (50 ng p24 per infection, $5 \times 10^{4}$ cells per well in 24 -well plates). 3T3.CD4 cells that stably express the different chemokine receptors ${ }^{2}$ were similarly infected. 3T3.CD4 cells expressing the new receptors were prepared by retroviral transduction with pMX.Bonzo and pMX.BOB. After 3 days, cells were resuspended in $120 \mu \mathrm{l}$ of luciferase lysis buffer (Promega). The luciferase activity in $20 \mu \mathrm{l}$ lysate was assayed in a Wallac Microbeta 1450 Counter using commercially available reagents (Promega).

Northern blots. Polyadenylated RNA was prepared from various human cell lines with the Micro-Fast Track kit (Invitrogen). Samples (5 $\mu$ g RNA) were electrophoresed through a $1 \%$ agarose-formaldehyde gel and transferred to a GeneScreen nitrocellulose membrane. Multiple-tissue northern blots I and II, purchased from Clontech, contain $\sim 2 \mu \mathrm{g}$ poly $(\mathrm{A})^{+}$RNA from each tissue. Integrity of blots was assayed by GAPDH probing. Full-length cDNAs of BOB and Bonzo were labelled with ${ }^{32} \mathrm{P}$ by using a Random Primed DNA-labelling kit (Boehringer-Mannheim) and used to probe northern blots.

Lymphocyte purification and RT-PCR. Monocytes were purified from buffy coats using a 46\% Percoll gradient. To purify T- and B-cell subsets, PBMC were stained with phycoerythrin-conjugated anti-CD3 or anti-CD19 antibodies (Becton-Dickinson) and sorted using FACS (Coulter). Total RNA was isolated using RNAzol reagent (Cinna/Biotecx), treated with RNase-free DNase, and $0.5 \mu \mathrm{g}$ was taken for cDNA synthesis using Superscript II RNAse H reverse transcriptase and random hexamer primers (Gibco-BRL); one-twentieth of this reaction was used as a template for PCR amplification with Taq DNA polymerase. BOB primers used for RT-PCR: upstream (from ATG) $5^{\prime}$ CATCTGCTCTTTGGTGATG; downstream (550 bp from ATG), 5' GTATGGCTTATCATCAATCAGC, amplifies $\sim 600 \mathrm{bp}$ of transcript; Bonzo primers were: upstream (from $270 \mathrm{bp}$ downstream of ATG), 5' -CAGGCATCCATGAATGGGTGT, and downstream (from the stop codon), 5'-CAAGGCCTATAACTGGAACATGCTG, amplifies $\sim 750 \mathrm{bp}$ of transcript. The PCR reaction was run for 30 cycles at $94^{\circ} \mathrm{C}$ for $40 \mathrm{~s}, 58^{\circ} \mathrm{C}$ for $40 \mathrm{~s}$, and $72^{\circ} \mathrm{C}$ for $1 \mathrm{~min}$. To exclude contamination of genomic DNA, control cDNA reactions in which reverse transcriptase was omitted were prepared in parallel. These were uniformly negative.

Received 27 May; accepted 23 June 1997.

1. Feng, Y., Broder, C. C., Kennedy, P. E. \& Berger, E. A. HIV-1 entry cofactor: functional cDNA cloning of a seven-transmembrane, G protein-coupled receptor. Science 272, 872-877 (1996).

2. Deng, H. et al. Identification of a major co-receptor for primary isolates of HIV-1. Nature 381, 661666 (1996).

3. Alkhatib, G. et al. CC CKR5: a RANTES, MIP-1 $\alpha$, MIP- $1 \beta$ receptor as a fusion cofactor fo macrophage-tropic HIV-1. Science 272, 1955-1958 (1996).

4. Dragic, T. et al. HIV-1 entry into $\mathrm{CD}^{+}$cells is mediated by the chemokine receptor CC-CKR-5. Nature 381, 667-673 (1996)

5. Doranz, B. J. et al. A dual-tropic primary HIV-1 isolate that uses fusin and the $\beta$-chemokine receptor CKR-5, CKR-3 and CKR-2b as fusion cofactors. Cell 85, 1149-1158 (1996).

6. Choe, H. et al. The beta-chemokine receptors CCR3 and CCR5 faciitate infection by primary HIV-1 isolates. Cell 85, 1135-1148 (1996)

7. Chen, Z., Zhou, P., Ho, D. D., Landau, N. R. \& Marx, P. A. Genetically divergent strains of simian immunodeficiency virus use CCR5 as a coreceptor for entry. J. Virol. 71, 2705-2715 (1997).

8. Marcon, L. et al. Utilization of C-C chemokine receptor 5 by the envelope glycoproteins of a pathogenic simian immunodeficiency virus, SIVmac239. J. Virol. 71, 2522-2527 (1997).

9. Edinger, A. L. et al. Differential utilization of CCR5 by macrophage and T cell tropic simian immunodeficiency virus strains. Proc. Natl Acad. Sci. USA 94, 4005-4010 (1997).

10. Hill, C. M. et al. Envelope glycoproteins from HIV-1, HIV-2, and SIV can use human CCR5 as coreceptor for viral entry and make direct CD4-dependent interactions with this chemokine receptor. J. Virol. (in the press).

11. Marthas, M. L. et al. Rhesus macaques inoculated with molecularly cloned simian immunodeficiency virus. J. Med. Primatol. 18, 311-319 (1989).

12. Fukasawa, M. et al. Sequence of simian immunodeficiency virus from African green monkey, a new member of the HIV/SIV group. Nature 333, 457-461 (1988).

13. Clapham, P. R., Blanc, D. \& Weiss, R. A. Specific cell surface requirements for the infection of CD4 positive cells by human immunodeficiency virus types 1 and 2 and by simian immunodeficiency virus. Virology 181, 703-715 (1991).

14. Stefano, K. A. et al. Replication of a macrophage-tropic strain of human immunodeficiency virus type $1(\mathrm{HIV}-1)$ in a hybrid cell line, $\mathrm{CEM} \times 174$, suggests that cellular accessory molecules are required for HIV-1 entry. J. Virol. 67, 6707-6715 (1993).

15. Heiber, M. et al. A novel human gene encoding a G-protein-coupled receptor (GPR15) is located on chromosome 3. Genomics 32, 462-465 (1996).

16. He, J. et al. CCR3 and CCR5 are co-receptors for HIV-1 infection of microglia. Nature 385, 645-649 (1997).

17. Fauci, A. S. Host factors and the pathogenesis of HIV-induced disease. Nature 384, 529-534 (1996) 18. Desrosiers, R. C. The simian immunodeficiency viruses. Annu. Rev. Immunol. 8, 557-578 (1990).

19. Kestler, H. et al. Induction of AIDS in rhesus monkeys by molecularly cloned simian immunodeficiency virus. Science 248, 1109-1112 (1990).

20. Theodorou, I., Meyer, L., Magierowska, M., Katlama, C. \& Rouzioux, C. HIV-1 infection in an individual homozygous for CCR5 delta 32. Seroco Study Group. Lancet 349, 1219-1220 (1997).

21. Biti, R. et al. HIV-1 infection in an individual homozygous for the CCR5 deletion allele. Nature Med. 3, 252-253 (1997)

22. O’Brien, T. R. et al. HIV-1 infection in a man homozygous for CCR5 delta 32. Lancet 349, 1219 (1997).
23. Kitamura, T. et al. Efficient screening of retroviral cDNA expression libraries. Proc. Natl Acad. Sci. USA 92, 9146-9150 (1995)

24. Landau, N. R., Page, K. A. \& Littman, D. R. Pseudotyping with human T-cell leukemia virus type I broadens the human immunodeficiency virus host range. J. Virol. 65, 162-169 (1991).

25. Westervelt, P., Gendelman, H. E. \& Ratner, L. Identification of a determinant within the human immunodeficiency virus 1 surface envelope glycoprotein critical for productive infection of primary monocytes. Proc. Natl Acad. Sci. USA 88, 3097-3101 (1991).

26. Hwang, S. S., Boyle, T. J., Lyerly, H. K. \& Cullen, B. R. Identification of envelope V3 loop as the major determinant of CD4 neutralization sensitivity of HIV-1. Science 257, 535-537 (1992).

27. Gao, F. et al. Molecular cloning and analysis of functional envelope genes from human immunodeficiency virus type 1 sequence subtypes A through G. (The WHO and NIAID Networks for HIV Isolation and Characterization.) J. Virol. 70, 1651-1657 (1996).

28. Barnett, S. W., Quiroga, M., Werner, A., Dina, D. \& Levy, J. A. Distinguishing features of an infectious molecular clone of the highly divergent and noncytopathic human immunodeficiency virus type 2 UC1 strain. J. Virol. 67, 1006-1014 (1993).

29. Kong, L. I. et al. West African HIV-2-related human retrovirus with attenuated cytopathicity. Science 240, 1525-1529 (1988)

30. Gao, F. et al. Human infection by genetically diverse SIVSM-related HIV-2 in west Africa. Nature 358, 495-499 (1992).

31. Liao, S. et al. J. Exp. Med. 185, 2015-2023 (1997).

Acknowledgements. We thank T. Kitamura for the pMX retroviral library; R. Sutton for construction of HIV-puro; C. Mackay for the monoclonal antibody against CCR5; P. Cresswell for 174 and CEM cell lines, B. Hahn for virus isolates; M. Emerman for pL-VSV-G; C. Weiss for HIV-2 Env vectors; G. Pare for technical assistance; W. Ellmeier for help with northern blots; and C. Davis, C. M. Hill and M. Vodicka for comments on the manuscript. This work was supported by grants from the NIH and by postdoctoral fellowships from the Aaron Diamond Foundation (H.-K.D.) and the Damon Runyon-Walter Winchell Foundation (V.N.K.). D.R.L. is an investigator of the Howard Hughes Medical Institute.

Correspondence and requests for materials should be addressed to D.R.L. (e-mail: littman@saturn.med. nyu.edu). Genbank sequence accession numbers are: human Bonzo, AF007545; BOB (African green monkey), AF007856; BOB (pigtailed macaque), AF007857; Bonzo (pigtailed macaque), AF007858; Bonzo (African green monkey), AF007859.

\section{X-linked IAP is a direct inhibitor of cell-death proteases}

\section{Quinn L. Deveraux ${ }^{\star}$, Ryosuke Takahashi Guy S. Salvesen \& John C. Reed}

The Burnham Institute, Program on Apoptosis and Cell Death Research, 10901 North Torrey Pines Road, La Jolla, California 92037, USA

* These authors contributed equally to this work.

The inhibitor-of-apoptosis (IAP) family of genes has an evolutionarily conserved role in regulating programmed cell death in animals ranging from insects to humans ${ }^{1-6}$. Ectopic expression of human IAP proteins can suppress cell death induced by a variety of stimuli, but the mechanism of this inhibition was previously unknown. Here we show that human X-chromosome-linked IAP directly inhibits at least two members of the caspase family of celldeath proteases, caspase- 3 and caspase-7. As the caspases are highly conserved throughout the animal kingdom and are the principal effectors of apoptosis ${ }^{7}$, our findings suggest how IAPs might inhibit cell death, providing evidence for a mechanism of action for these mammalian cell-death suppressors.

For our initial investigation of human X-chromosome-linked IAP (XIAP; hILP; MIHA), we used recombinant XIAP protein and a cell-free system in which cytochrome $c$ was added to cytosolic extracts. When added to normal cytosol, cytochrome $c$ initiates an apoptotic programme which includes proteolytic processing and activation of certain caspases, and apoptotic-like destruction of exogenously added nuclei ${ }^{8}$. Release of cytochrome $c$ from mitochondria into the cytosol appears to be commonly associated with apoptosis $^{8,9}$.

Purified nuclei mostly remained intact in the cytosol from controls. In contrast, adding cytochrome $c$ caused apoptotic-like destruction of nearly all nuclei (Fig. 1A). Addition of cytochrome $c$ directly to nuclei without cytosol had no effect, demonstrating the requirement for cytosolic factors (results not shown) ${ }^{8}$. Recombinant XIAP (rXIAP) added simultaneously with cytochrome $c$ severely inhibited nuclear destruction (Fig. 1A). An equivalent 